\title{
Carpal tunnel syndrome
}

\section{Victoria Squissato MSc HBSc, Glenn Brown MD MPH}

\section{Carpal tunnel syndrome may be less frequent among people working in high-risk manufacturing and office settings than is commonly thought}

A prospective long-term study of a general working-age population (16-74 yr) showed that the overall incidence of carpal tunnel syndrome over a 66-month period was 103 per 100000 people per year (95\% confidence interval [CI] 87119), based on a clinical definition (i.e., clinical symptoms and signs). ${ }^{1}$ Among the high-risk occupational groups, "skilled trades" and "administrative and secretarial," the incidences were 136 (95\% CI 115-158) and 82 (95\% CI 64-99) per 100000 people per year, respectively. ${ }^{1}$

The association between carpal tunnel syndrome and use of a computer keyboard or mouse is unclear

A systematic review of eight epidemiologic studies concluded that because of bias, lack of consistency and poor statistical power, the evidence is insufficient to confirm that computer work causes carpal tunnel syndrome. ${ }^{3}$ The review also found no evidence of an association with repetitive, low-force noncomputer work. ${ }^{3}$ However, a more recent cross-sectional study that calculated the cumulative number of keyboard strokes per person per year, using employee data from a payroll registry, found that a high level of exposure (at least 240.5 million strokes per year) increased the risk of carpal tunnel syndrome (OR 2.23, 95\% CI 1.09-4.52). ${ }^{4}$ Given that epidemiologic studies of occupational association are subject to limitations, additional studies are warranted.

For references, please see Appendix 1, available at www.cmaj.ca/lookup/suppl /doi:10.1503/cmaj.131177/-/DC1
Personal characteristics that increase a patient's risk include age, sex and obesity

A two-year cross-sectional study of tradespeople, professionals and computer workers found that, per 10-year increase in age, the OR of carpal tunnel syndrome increased by 1.55 (95\% CI 1.31-1.86). Women were at higher risk than men (OR 2.24, 95\% CI 1.43$3.50)$, and the OR increased by 1.32 (95\% CI 1.15-1.52) per 5-point increase in body mass index. ${ }^{5}$

Competing interests: The Ontario Workplace Safety and Insurance Board's Champions Educational Grant to Queen's University supported a summer studentship for Victoria Squissato.

This article has been peer reviewed.

Affiliations: School of Medicine (Squissato), Department of Family Medicine (Brown), Department of Anesthesiology and Perioperative Medicine (Brown), Queen's University, Kingston, Ont.

Correspondence to: Glenn Brown, glenn.brown@dfm.queensu.ca

CMAJ 2014. DOI:10.1503/cmaj.131177
Occupational exposure to excess vibration, increased hand force and repetition increase the risk of carpal tunnel syndrome

Using a conservative definition that included nerve conduction abnormalities with symptoms and signs consistent with carpal tunnel syndrome, a meta-analysis of 37 studies found that factors significantly associated with increased risk included vibration (odds ratio [OR] 5.40, 95\% CI 3.14-9.31), increased hand force (OR 4.23, 95\% CI 1.53-11.68) and repetition (OR 2.26, 95\% CI 1.73-2.94). ${ }^{2}$

Initial management may include occupational therapy and wrist splinting

In a recent study, patients on a waiting list for carpal tunnel release surgery were randomly assigned to either eight weeks of splinting and education sessions with an occupational therapist or no treatment. ${ }^{6}$ Symptom severity, functional status, pain perception and grip strength all improved in the intervention group. ${ }^{6}$ Moreover, at the end of the study period, only $37 \%$ of the intervention group decided to pursue surgical treatment. ${ }^{6}$ If symptoms do not improve within eight weeks, referral to a surgical specialist (orthopedics or plastics) should be considered.

\section{Physician resource}

A self-directed learning module on occupational medicine and carpal tunnel syndrome created by the authors may be freely accessed at https://meds.queensu.ca/central/comm unity/carpaltunnelsyndrome 